\title{
Mulinum ulicinum (Azorelloideae, Apiaceae), nueva cita para Chile
}

\section{Mulinum ulicinum (Azorelloideae, Apiaceae), new record for Chile}

\author{
Martina Fernández ${ }^{*}$ Cecilia Ezcurra \& Carolina I. Calviño \\ Instituto de Investigaciones en Biodiversidad y Medioambiente, INIBIOMA, CONICET-Universidad Nacional del Comahue, \\ Quintral 1250, 8400 San Carlos de Bariloche, Río Negro, Argentina. \\ *mfernandez@comahue-conicet.gob.ar
}

\begin{abstract}
Mulinum ulicinum is recorded for the first time in Chile. This species and M. crassifolium are the only two species of the genus growing in the Region of Antofagasta. A description and illustration of M. ulicinum is provided, as well as a key to differentiate it from M. crassifolium.
\end{abstract}

El género Mulinum Pers. es sudamericano, característico de la estepa patagónica y de regiones elevadas de los Andes australes. Se extiende por Chile, sur de Bolivia y Argentina desde el nivel del mar hasta los $4.900 \mathrm{~m}$ de altitud. Consta de 13 especies que crecen en terrenos arenosos, roquedales y pedreros áridos tanto en la estepa como en la alta montaña. En Chile, el género incluye seis especies, de las cuales sólo $M$. crassifolium Phil. estaba citada para el norte chileno (regiones de Antofagasta y Atacama; Martínez 2008). En el género Mulinum existen varias especies de difícil determinación porque son muy afines morfológicamente y porque sus áreas de distribución se superponen.

Mulinum ulicinum Gillies \& Hook. fue descrita para Argentina y su presencia en Chile se consideraba dudosa (Martínez 2008). Sin embargo, la hemos coleccionado tanto del lado argentino como chileno a la altura del Paso de Jama que une Jujuy con la Región de Antofagasta, confirmando su presencia en Chile. Mulinum ulicinum ha sido, sin embargo, ampliamente recolectada en Chile aunque mal determinada en herbarios como M. crassifolium.

En esta contribución se presenta una descripción e ilustración de M. ulicinum, y se proporciona una clave para diferenciarla de $M$. crassifolium, la especie con la que vive simpátricamente en la Región de Antofagasta y con la que ha sido confundida.

\section{DESCRIPCIÓN}

Mulinum ulicinum Gillies \& Hook., Bot. Misc. 1: 328, t. 64. 1830. TIPO: Argentina. Mendoza: montañas de Uspallata. Gillies s.n. (holotipo, K no visto [foto!]; isotipo, E no visto [foto!]). Fig. 1.

Subarbusto androdioico que forma matas bajas, irregulares, laxas, resinosas, de 5-30 cm de altura y $15-30 \mathrm{~cm}$ de diámetro. Hojas de 6-23 mm, pecioladas, obovadas, rígidas, lámina de
3-11 x 4-12 mm, glabra, 3-partida; segmentos triangulares a angostamente triangulares, aciculares, surcados en la cara adaxial, el central 2-11 x 0,3-1,4 mm, margen entero, laterales similares al central, margen entero; ápice agudo, espinoso, punzante; pecíolo delgado, semejante a los segmentos, de 2-10 mm, glabro; base foliar apenas ensanchada, membranácea, margen entero o escasamente ciliado. Umbelas simples, terminales, muy por debajo del nivel de las hojas, con 2-6 flores perfectas o 7-15 flores estaminadas; pedúnculos de 2,5-7 $\mathrm{mm}$; involucro de 2-6 brácteas de 2-6 mm, adpresas, connadas hasta la mitad o sólo en la base, formando una cúpula con lóbulos anchamente triangulares, márgenes enteros, glabros. Pedicelos florales de 2-6 mm; pétalos amarillos, ovados, de 1,5-2 x 1-1,8 mm; estilopodio deprimido a algo cónico, estilos de 1-1,2 mm. Fruto esquizocarpo amarillo a rojo, de contorno elíptico, muy comprimido dorsalmente, de 3-5 x 2-4 mm y 1-1,5 $\mathrm{mm}$ de espesor cada mericarpio, alas más angostas que el cuerpo del fruto, de 0,5-1 mm de latitud; carpóforo libre, entero o cortamente bífido, los mericarpios se separan fácilmente.

DisTRIBUCIÓN, HÁBITAT Y FENOLOGÍA

El área de distribución de esta especie incluye Argentina (Jujuy, Salta, San Juan y Mendoza) y Chile (Región de Antofagasta). Se encuentra en cumbres, laderas altas, y abras planas y expuestas entre rocas desde 2.900-4.900 $\mathrm{m}$ de altitud. Florece de diciembre a marzo. Fructifica de diciembre a abril.

\section{OBSERVACIONES}

Martínez (2008) consideró dudosa la presencia de M. ulicinum en Chile, probablemente a partir de los tratamientos realizados por Gay (1848-1849), Weddell (1857) y Reiche (1902) sobre la flora de Chile, en los 
que se la incluye. Sin embargo, tal como aclararemos, las citas de M. ulicinum en Chile por estos autores se debieron a incorrectas determinaciones de la especie.

Gay (1848-1849) describió a M. ulicinum sinonimizándolo con $M$. echinus DC. En su tratado, no citó material examinado, pero seguramente se basó en el material tipo de $M$. ulicinum recolectado por Gillies en Uspallata, Mendoza, Argentina, y en el material tipo de M. echinus coleccionado por Haenke en Chile (Fernández et al., en preparación). De allí que indicó que M. ulicinum habitaba las cordilleras que median entre Santiago y Mendoza. Por lo tanto, la cita de Gay (1848-1849) de $M$. ulicinum en Chile, en realidad corresponde a M. echinus, ya que estas especies hoy no se consideran sinónimos. Más aún, luego de una exhaustiva revisión de M. ulicinum en herbarios, no hemos encontrado ningún ejemplar de esta especie coleccionado en Chile a la altura de Santiago, y tampoco la encontramos recorriendo la zona. En forma análoga, Weddell (1857) describió e ilustró un ejemplar de M. spinosum (Cav.) Pers. y lo denominó equivocadamente como M. ulicinum. Por último, Reiche (1902) trató a $M$. ulicinum erróneamente como una variedad de $M$. spinosum en base a las observaciones ya hechas por Gay (1848-1849) y Weddell (1857).

Mulinum ulicinum se distingue fácilmente de $M$. echinus y M. spinosum. Estas dos últimas especies forman grandes cojines hemisféricos, presentan pedúnculos florales largos que en general sobrepasan el nivel de las hojas, y poseen frutos anchamente alados. En cambio, M. ulicinum forma matas bajas, presenta pedúnculos florales cortos que no sobrepasan las hojas, y posee frutos casi ápteros.
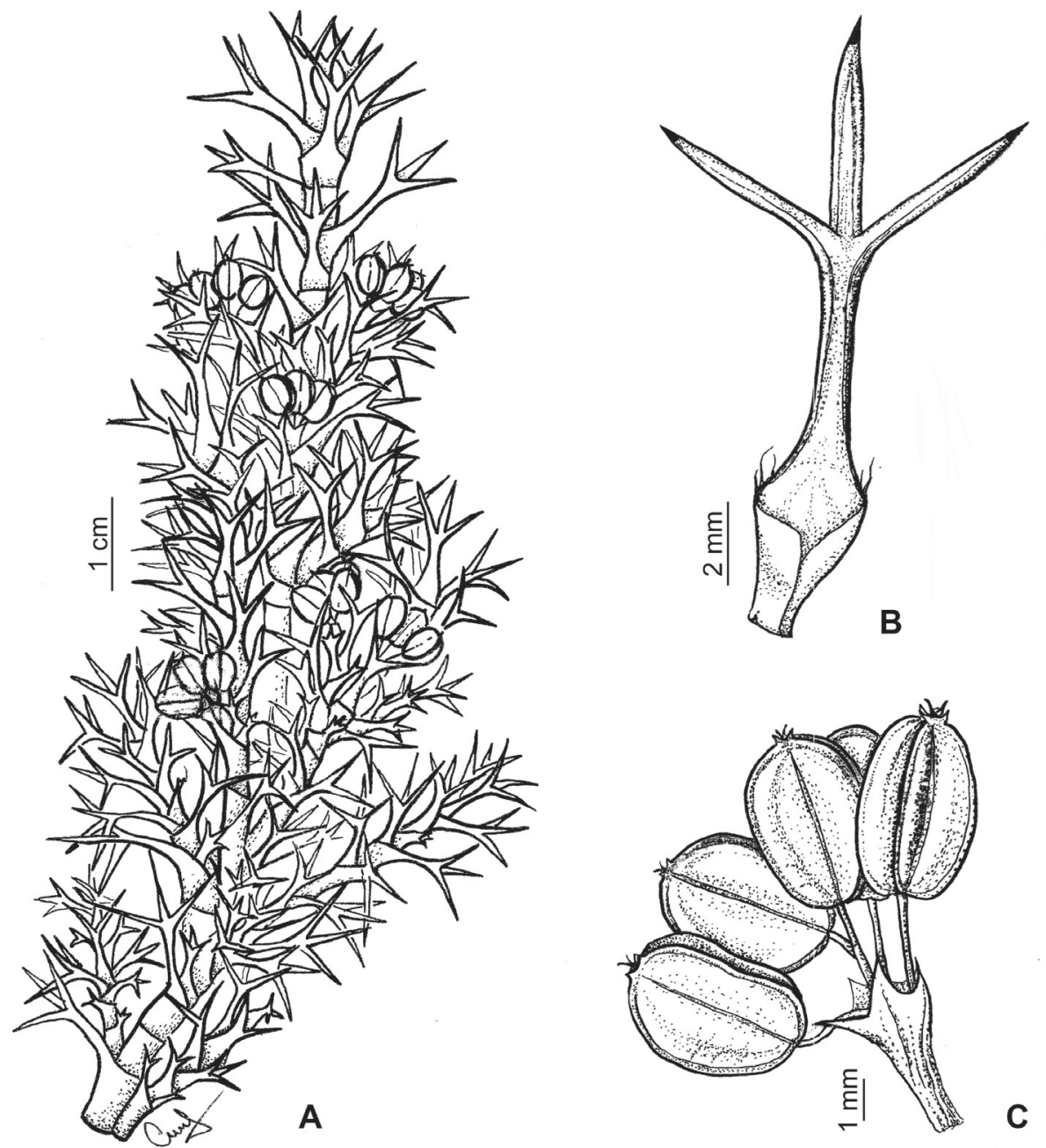

Figura 1. Mulinum ulicinum. A. Aspecto general. B. Hoja. C. Umbela con frutos (Ruiz Leal \& Roig 23635, MERL).

Figure 1. Mulinum ulicinum. A. Habit. B. Leaf. C. Umbel with fruits (Ruiz Leal \& Roig 23635, MERL). 
Material estudiado

CHILE, Región de Antofagasta, Prov. El Loa, a $10 \mathrm{~km}$ del límite con Argentina, 4.430 m. 14-XII-2010, Calviño et al. 792 (BCRU); cerro Curutú lado S del Paso de Jama, 4.700 m. IV-1997, Arroyo et al. 97268 (CONC); cerro nevados de Poquis, ladera SO, 4.500 m. IV-1997, Arroyo et al. 97385 (CONC); cordón Ceja Alta, lado SE, 4.600 m. IV-1997, Arroyo et al. 97290 (CONC); borde sur del Salar de Tara, 4.120 m. 18-XII-1996, Moreira \& Muñoz 300 (CONC); cruce entre Paso de Jama y Salar de Tara, 4.250 m. 19-XII1996, Moreira \& Muñoz 318 (CONC).

Material ARGENTINo DE REFERENCIA

ARGENTINA, Prov. Jujuy, Dpto. Humahuaca, Mina Aguilar, camino al campamento viejo, 4.400 m. 16-XII2010, Calviño et al. 797 (BCRU). Dpto. Rinconada, Campo Lagunilla, camino hacia Aparzo, 4.100 m. 7-II-1979, Gonzalez 22 (LIL). Dpto. San Antonio de los Cobres, Qda. de Polvorillas, 4.200 m. 2-II-1944, Cabrera 8353 (LP). Dpto. Susques, unos kms después del mirador del salar de
Jama, 4.250 m. 14-XII-2010, Calviño et al. 793 (BCRU). Dpto. Tumbaya, El Moreno, 3.500 m. 3-II-1901, Fries 840 (CORD). Prov. Mendoza, Dpto. Las Heras, Alto de los Manantiales, 2.900-3.100 m. 20-II-1965, Roig 5234 (MERL); Dpto. Malargüe, Malargüe, entre Ao. Chaguitracó y Portezuelo del Choique, 16-I-1958, Ruiz Leal \& Roig 18833 (MERL). Dpto. Luján de Cuyo, zona del cerro del Plata: Piedra Grande, 3.600-3.700 m. 12-I-1960, Ruiz Leal 21045 (MERL). Prov. Salta, Dpto. Coronel Moldes, Mina Concordia, Gobernación de los Andes, 4.500 m. 01-X-1929, Mintzer 60102 (BAB). Dpto. La Poma, Abra de Acay, 4.895 m. 18-II-1986, Varela \& Del Castillo 1362 (MCNS). Dpto. Los Andes, camino a Alto de Tocomar, km 181 de la RN51, 4.500 m. 17-XII-2010, Calviño et al. 798 (BCRU). Prov. San Juan, Dpto. Calingasta, Río Manrique a Portezuelo de Potrerillos, 3.000 m. 23-I-1991, Kiesling 7688 (SI); SO de Barreal, zona de El Mercedario, cordón de la Ramada, 3.200 m. 30-XII-2010, Kiesling 10408 (BCRU, MERL). Dpto. Iglesia, Cordillera de Colangüil: de Invernadas a la Quebrada del Salto, 15-I-1930, Pérez Moreau 30/147 (BA).

Clave Para diferenciar a Mulinum ulicinum de M. CRASSifolium

1. Matas irregulares. Hojas pecioladas, no crasas, segmentos triangulares a angostamente triangulares, aciculares. Ápice de las hojas agudo, espinoso y punzante. Fruto de contorno elíptico, muy comprimido dorsalmente, alas más angostas que el cuerpo del fruto. Carpóforo libre, los mericarpios se separan fácilmente............................................................................................... ulicinum

1'. Cojines hemisféricos. Hojas cortamente pecioladas, crasas, segmentos muy anchamente ovados a anchamente ovados, planos. Ápice de las hojas muy obtuso a redondeado, mucronado y no punzante. Fruto de contorno anchamente obovado, poco comprimido dorsalmente, alas igual o más anchas que el cuerpo del fruto. Carpóforo adherido a los mericarpios, los mericarpios se separan con dificultad.

M. crassifolium

\section{AGRADECIMIENTOS}

A los curadores de los herbarios citados por el envío de ejemplares, a Ailén Santomé por la ilustración, y a CONICET PIP-00282, ANPCyT-FONC y T-PICT-2011-1036, y UNComahue PIN-B180 por el apoyo económico. También agradecemos a dos revisores anónimos por los comentarios al manuscrito.

\section{BIBLIOGRAFÍA}

GAY, C. 1848-1849. Botánica. Historia Física y Política de Chile. Umbelíferas. Vol. 3, pp. 61-145. Museo de Historia Natural de Santiago, Santiago, Chile.

Martínez, S. 2008. Apiaceae. En: F.O. Zuloaga, O. Morrone \& M. Belgrano (eds.), Catálogo de las Plantas Vasculares del Cono Sur, Vol. 2, pp. 1056-1090. Monographs in Systematic Botany from the Missouri Botanical Garden, Missouri, USA.

ReIche, K. 1902. Flora de Chile. Umbelíferas. Vol. 3, pp. 46-121. Santiago, Chile.

Weddell, H.A. 1857. Chloris Andina. Umbelliferae. Vol. 2, pp. 186-206. Bertrand, Paris, Francia.

Recibido: 11.03.14

Aceptado: 04.06.14 